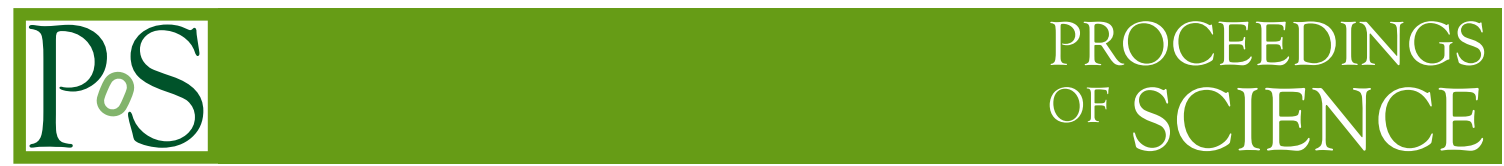

\title{
Recent results on Kaon physics
}

\author{
M. Antonelli* \\ Laboratori Nazionali di Frascati dell'INFN, Frascati, Italy \\ E-mail: Mario.Antonelli@lnf.infn.it
}

Recent results on Kaon physics are summarized. Progress in the determination of direct and indirect CP violation parameters and improvements in the CPT invariance are reported. The special role of helicity suppressed modes and their sensitivity to new-physics is discussed.

Sixth International Workshop on Chiral Dynamics

July 6-10 2009

Bern, Switzerland

* Speaker. 


\section{A test of CPT invariance}

Within the Wigner-Weisskopf approximation, the time evolution of the neutral kaon system is described by [1]

$$
i \frac{\partial}{\partial t} \Psi(t)=H \Psi(t)=\left(M-\frac{i}{2} \Gamma\right) \Psi(t)
$$

where $M$ and $\Gamma$ are $2 \times 2$ time-independent Hermitian matrices and $\Psi(t)$ is a two-component state vector in the $K^{0}-\bar{K}^{0}$ space. Denoting by $m_{i j}$ and $\Gamma_{i j}$ the elements of $M$ and $\Gamma$ in the $K^{0}-\bar{K}^{0}$ basis, $C P T$ invariance implies

$$
m_{11}=m_{22} \quad\left(\text { or } m_{K^{0}}=m_{\bar{K}^{0}}\right) \quad \text { and } \quad \Gamma_{11}=\Gamma_{22} \quad\left(\text { or } \Gamma_{K^{0}}=\Gamma_{\bar{K}^{0}}\right) .
$$

The eigenstates of eq. (1.1) can be written as

$$
\begin{aligned}
K_{S, L} & =\frac{1}{\sqrt{2\left(1+\left|\varepsilon_{S, L}\right|^{2}\right)}}\left(\left(1+\varepsilon_{S, L}\right) K^{0} \pm\left(1-\varepsilon_{S, L}\right) \bar{K}^{0}\right), \\
\varepsilon_{S, L} & =\frac{-i \operatorname{Im}\left(m_{12}\right)-\frac{1}{2} \operatorname{Im}\left(\Gamma_{12}\right) \pm \frac{1}{2}\left(m_{\bar{K}^{0}}-m_{K^{0}}-\frac{i}{2}\left(\Gamma_{\bar{K}^{0}}-\Gamma_{K^{0}}\right)\right)}{m_{L}-m_{S}+i\left(\Gamma_{S}-\Gamma_{L}\right) / 2} \\
& \equiv \varepsilon \pm \delta,
\end{aligned}
$$

such that $\delta=0$ in the limit of exact $C P T$ invariance. Unitarity allows us to express the four measurements of $\Gamma$ in terms of appropriate combinations of the kaon decay amplitudes $\mathscr{A}_{i}$ :

$$
\Gamma_{i j}=\sum_{f} \mathscr{A}_{i}(f) \mathscr{A}_{j}(f)^{*}, \quad i, j=1,2=K^{0}, \bar{K}^{0},
$$

where the sum runs over all the accessible final states. Using this decomposition in eq. (1.4) leads to the Bell-Steinberger relation (BSR): a link between $\operatorname{Re}(\varepsilon), \operatorname{Im}(\delta)$, and the physical kaon decay amplitudes. In particular, without any expansion in the $C P T$-conserving parameters and neglecting only $\mathscr{O}(\varepsilon)$ corrections to the coefficient of the $C P T$-violating parameter $\delta$, one finds

$$
\left(\frac{\Gamma_{S}+\Gamma_{L}}{\Gamma_{S}-\Gamma_{L}}+i \tan \phi_{\mathrm{SW}}\right)\left(\frac{\operatorname{Re}(\varepsilon)}{1+|\varepsilon|^{2}}-i \operatorname{Im}(\delta)\right)=\frac{1}{\Gamma_{S}-\Gamma_{L}} \sum_{f} \mathscr{A}_{L}(f) \mathscr{A}_{S}^{*}(f)
$$

where $\phi_{\mathrm{SW}}=\arctan \left(2\left(m_{L}-m_{S}\right) /\left(\Gamma_{S}-\Gamma_{L}\right)\right)$.

The advantage of the $K^{0}-\overline{K^{0}}$ system with respect to the $D^{0}-\overline{D^{0}}$ and $B^{0}-\overline{B^{0}}$ systems is that only a few decay modes give significant contributions to the r.h.s. in eq. (1.6): only the $\pi \pi(\gamma), \pi \pi \pi$ and $\pi \ell v$ modes turn out to be relevant up to the $10^{-7}$ level.

Recent measurements of neutral Kaon paramametres from KLOE, $\mathrm{KTeV}$, and NA48, provided a new improved set of inputs to eq 1.6. A summary of the present situation is described in the Review of Particle Physics [2]. The values of $\operatorname{Re}(\varepsilon)$ and $\operatorname{Im}(\delta)$ determined using the world average of published results are:

$$
\begin{aligned}
& \operatorname{Re}(\varepsilon)=(161.2 \pm 0.6) \times 10^{-5} \\
& \operatorname{Im}(\delta)=(-0.6 \pm 1.9) \times 10^{-5}
\end{aligned}
$$


The accuracy of this test is limited by the knowledge on the parameters describing the two-pion final states:

$$
\frac{1}{\Gamma_{S}}\left\langle\mathscr{A}_{L}(i) \mathscr{A}_{S}^{*}(i)\right\rangle=\eta_{i} \mathrm{BR}\left(K_{S} \rightarrow i\right), \quad i=\pi^{0} \pi^{0}, \pi^{+} \pi^{-}(\gamma),
$$

The $\eta_{i}$ parameters in eq. (1.9) are the usual amplitude ratios: $\eta_{i}=\mathscr{A}_{L}(i) / \mathscr{A}_{S}(i)$.

The recent prelimary KTeV analysis improves the determination of the phases of $\eta_{i}$ leading to:

$$
\begin{aligned}
& \operatorname{Re}(\varepsilon)=(161.2 \pm 0.6) \times 10^{-5} \\
& \operatorname{Im}(\delta)=(-0.6 \pm 1.4) \times 10^{-5}
\end{aligned}
$$

\section{Indirect and direct $\mathrm{CP}$ violation}

$C P$ violation was discovered in 1964 through the observation of the decay $K_{L} \rightarrow \pi^{+} \pi^{-}$[3]. Indirect CP violation parameters is nowdays best determined from measurements of the same process. The value of $\operatorname{BR}\left(K_{L} \rightarrow \pi^{+} \pi^{-}\right)$is known today with high accuracy from the new results by KLOE [4], KTeV [5], and NA48 [6].

In the Standard Model, $C P$ violation is naturally accommodated by a phase in the quark mixing matrix $[9,10] . \mathrm{BR}\left(K_{L} \rightarrow \pi^{+} \pi^{-}\right)$, together with the well known values of $\mathrm{BR}\left(K_{S} \rightarrow \pi^{+} \pi^{-}\right), \tau_{K_{S}}$, and $\tau_{K_{L}}$, determines the modulus of the amplitude ratio $\left|\eta_{+-}\right|=$ $\sqrt{\Gamma\left(K_{L} \rightarrow \pi^{+} \pi^{-}\right) / \Gamma\left(K_{S} \rightarrow \pi^{+} \pi^{-}\right)}$, which is related to the $C P$ violation parameters $\varepsilon$ and $\varepsilon^{\prime}$ by $\eta_{+-}=\varepsilon+\varepsilon^{\prime} \simeq \varepsilon$ [7]. The new experimental set gives $\left|\varepsilon^{\text {exp }}\right|=(2.223 \pm 0.006) \times 10^{-3} 1$.

The value of $|\varepsilon|$ determined above can compared with the Standard Model calculation:

$$
|\varepsilon|=C_{\varepsilon} \hat{B}_{K} A^{2} \lambda^{6} \bar{\eta}\left\{-\eta_{1} S_{0}\left(x_{c}\right)\left(1-\frac{\lambda^{2}}{2}\right)+\eta_{3} S_{0}\left(x_{c}, x_{t}\right)+\eta_{2} S_{0}\left(x_{t}\right) A^{2} \lambda^{4}(1-\bar{\rho})\right\},
$$

In Eq. (2.1) the Inami-Lim functions $S_{0}\left(x_{c, t}\right)$ and $S_{0}\left(x_{c}, x_{t}\right)$ [11] contain the box-contributions from the charm and top-quark exchange with $x_{i}=m_{i}^{2} / M_{W}^{2}$, while $\eta_{i}(i=1,2,3)$ describe (perturbative) short-distance QCD-corrections [12-14]. The Kaon bag parameter $B_{K}$ measures the deviation of the $\Delta S=2$ hadronic matrix element from its value in the vacuum-saturation approach.

Currently the best determination of this parameter is available from lattice simulations of QCD with either $2+1$ or 2 dynamical quark flavors. At present, the most accurate results (obtained independently with $2+1$ dynamical quark flavors) by RBC/UKQCD collaboration [15] and by Aubin et al. [16] quote a total uncertainty (statistical and systematic errors combined) of 5.4 and 4.0 per cent for $B_{K}$, respectively. Therefore, the contribution from $B_{K}$ to the total uncertainty in $\varepsilon$ is now comparable to the second biggest contribution, which originates from $V_{c b}$. This CKM-matrix element is nowadays known with 2.3 per cent accuracy [2] but enters $\varepsilon$ in the fourth power.

Many theoretical predictions of $\varepsilon^{t h}$ within the SM are available, showing only a mild agreement with the experimental determination. The difference $\left(\varepsilon^{\exp }-\varepsilon^{t h}\right) / \sigma_{\varepsilon}$ ranges between 1.8 and 2.1, depending on the inputs used for the epsilon ${ }^{\text {th }}$ determination.

\footnotetext{
${ }^{1}$ The difference with respect to the average of measurements performed before $2004, \varepsilon=(2.284 \pm 0.014) \times 10^{-3}$ [8], can be ascribed to a better treatment of radiative corrections in most recent measurements.
} 
Direct CP violation through $\Delta S=1$ transitions has been measured as a tiny difference in the normalized branching ratios of the $K_{L}$ to the CP-even eigenstates, $K_{L} \rightarrow \pi^{+} \pi^{-}$and $K_{L} \rightarrow \pi^{0} \pi^{0}$. The ratio $\varepsilon^{\prime} / \varepsilon$ is determined from:

$$
\left|\frac{\eta_{00}}{\eta_{+-}}\right|^{2} \simeq 1-6 \operatorname{Re}\left(\frac{\varepsilon^{\prime}}{\varepsilon}\right)
$$

Recentely the $\mathrm{KTeV}$ collaboration has presented new results from an analysis of their full data sample. The analysis includes many improvements in charged and neutral event reconstruction and simulation. In particular, the calibration of the CSI calorimeter has been improved substantially leading to a factor of 2 reduction in the related systematic uncertainty. They found:

$$
\operatorname{Re}\left(\frac{\varepsilon^{\prime}}{\varepsilon}\right)=(19.2 \pm 1.1(\text { stat }) \pm 1.8(\text { syst })) \times 10^{-4}
$$

in agreement with the NA48 result $\operatorname{Re}\left(\frac{\varepsilon^{\prime}}{\varepsilon}\right)=(14.7 \pm 2.2) \times 10^{-4}$. The world average, including the old NA31 and E731 result, is $\operatorname{Re}\left(\frac{\varepsilon^{\prime}}{\varepsilon}\right)=(16.8 \pm 1.4) \times 10^{-4}$.

\section{Tests of new-physics effects with helicity suppressed modes}

Helicity suppressed processes, like $K \rightarrow \ell v$, are one of the best indirect probes for new physics. For example, in two Higgs doublet models of type-II, such as the Higgs sector of the MSSM, sizeable contributions are potentially generated by charged-Higgs exchange diagrams (see e.g. Ref. [17-19]).

A particularly interesting test is the comparison of the $\left|V_{u s}\right|$ value extracted from the helicitysuppressed $K_{\ell 2}$ decays with respect to the value extracted from the helicity-allowed $K_{\ell 3}$ modes. To reduce theoretical uncertainties from $f_{K}$ and electromagnetic corrections in $K_{\ell 2}$, we exploit the ratio $B r\left(K_{\ell 2}\right) / B r\left(\pi_{\ell 2}\right)$ and we study the quantity

$$
R_{l 23}=\left|\frac{V_{u s}\left(K_{\ell 2}\right)}{V_{u s}\left(K_{\ell 3}\right)} \times \frac{V_{u d}\left(0^{+} \rightarrow 0^{+}\right)}{V_{u d}\left(\pi_{\ell 2}\right)}\right| .
$$

Within the SM, $R_{l 23}=1$, while deviation from 1 can be induced by non-vanishing scalar- or righthanded currents. Notice that in $R_{l 23}$ the hadronic uncertainties enter through $\left(f_{K} / f_{\pi}\right) / f_{+}(0)$.

Effects of scalar currents due to a charged Higgs give

$$
R_{l 23}=\left|1-\frac{m_{K^{+}}^{2}}{M_{H^{+}}^{2}}\left(1-\frac{m_{d}}{m_{s}}\right) \frac{\tan ^{2} \beta}{1+\varepsilon_{0} \tan \beta}\right|
$$

In the case of scalar densities (MSSM), the unitarity relation between $\left|V_{u d}\right|$ extracted from $0^{+} \rightarrow 0^{+}$nuclear beta decays and $\left|V_{u s}\right|$ extracted from $K_{\ell 3}$ remains valid as soon as form factors are experimentally determined. In this scenario,

$$
\left.R_{l 23}\right|_{\text {scalar }} ^{\exp }=1.004 \pm 0.007 .
$$

Here $\left(f_{K} / f_{\pi}\right) / f_{+}(0)$ has been fixed from lattice. This ratio is the key quantity to be improved in order to reduce present uncertainty on $R_{l 23}$. 


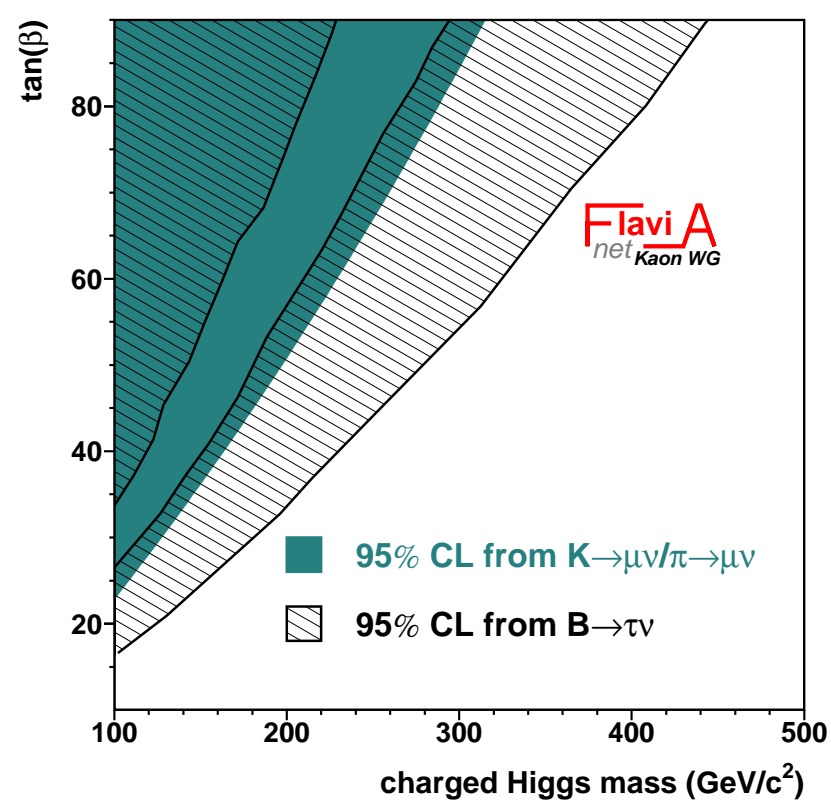

Figure 1: Excluded region in the charged Higgs mass-tan $\beta$ plane. The region excluded by $B \rightarrow \tau \nu$ is also indicated.

The measurement of $R_{l 23}$ above can be used to set bounds on the charged Higgs mass and $\tan \beta$. Figure 1 shows the excluded region at $95 \% \mathrm{CL}$ in the $M_{H}-\tan \beta$ plane (setting $\varepsilon_{0}=0.01$ ). The measurement of $\operatorname{BR}(B \rightarrow \tau v)$ [20] can be also used to set a similar bound in the $M_{H}-\tan \beta$ plane. While $B \rightarrow \tau \nu$ can exclude quite an extensive region of this plane, there is an uncovered region in the exclusion plot, which corresponds to a destructive interference between the chargedHiggs and the SM amplitude. This region is fully covered by the $K \rightarrow \mu v$ result.

\subsubsection{Lepton universality tests in $K_{\ell 2}$ decays}

The decay $K^{ \pm} \rightarrow e^{ \pm} v$ is strongly suppressed, $\sim$ few $\times 10^{-5}$, because of conservation of angular momentum and the vector structure of the charged weak current. It therefore offers the possibility of detecting minute contributions from physics beyond the SM. This is particularly true of the ratio $R_{K}=\Gamma(K \rightarrow e v) / \Gamma(K \rightarrow \mu v)$ which, in the SM, is calculable without hadronic uncertainties [21,22].

Recently it has been pointed out that in a supersymmetric framework sizable violations of lepton universality can be expected in $K_{l 2}$ decays [19]. At the tree level, lepton flavor violating terms are forbidden in the MSSM. However, these appear at the one-loop level, where an effective $H^{+} l v_{\tau}$ Yukawa interaction is generated. Following the notation of Ref. [19], the non-SM contribution to $R_{K}$ can be written as

$$
R_{K}^{L F V} \approx R_{K}^{S M}\left[1+\left(\frac{m_{K}^{4}}{M_{H^{ \pm}}^{4}}\right)\left(\frac{m_{\tau}^{2}}{m_{e}^{2}}\right)\left|\Delta_{13}\right|^{2} \tan ^{6} \beta\right]
$$


The lepton flavor violating coupling $\Delta_{13}$, being generated at the loop level, could reach values of $\mathscr{O}\left(10^{-3}\right)$. For moderately large $\tan \beta$ values, this contribution may therefore enhance $R_{K}$ by up to a few percent. Since the additional term in Eq. 3.4 goes with the forth power of the meson mass, no similar effect is expected in $\pi_{l 2}$ decays.

$R_{K}$ is defined to be inclusive of IB, ignoring however DE contributions. A recent calculation [22], which includes order $e^{2} p^{4}$ corrections in chiral perturbation theory gives:

$$
R_{K}=(2.477 \pm 0.001) \times 10^{-5} .
$$

$R_{K}$ is not directly measurable, since IB cannot be distinguished from DE on an event-by-event basis. Therefore, in order to compare data with the SM prediction at the percent level or better, the DE contribution must be carefully estimated and subtracted. ${ }^{2}$

DE can proceed through vector and axial-vector transitions, with effective coupling $V$ and $A$, respectively:

$$
\begin{aligned}
& \frac{\mathrm{d}^{2} \Gamma\left(K_{e 2 \gamma}, \mathrm{DE}\right)}{\mathrm{d} x d y}=\frac{G_{F}^{2}\left|\sin \theta_{\mathrm{C}}\right|^{2} \alpha_{\mathrm{em}} M_{K}^{5}}{64 \pi^{2}} \times \\
& {\left[(V+A)^{2} f_{\mathrm{DE}^{+}}(x, y)+(V-A)^{2} f_{\mathrm{DE}^{-}}(x, y)\right],}
\end{aligned}
$$

where $G_{F}$ is the Fermi coupling, $\theta_{\mathrm{C}}$ is the Cabibbo angle, $x=2 E_{\gamma} / M_{K}, y=2 E_{e} / M_{K}$ are the dimensionless photon and electron energies in the kaon rest frame (both lying between 0 and 1), and

$$
\begin{aligned}
& f_{\mathrm{DE}^{+}}(x, y)=(x+y-1)^{2}(1-x), \\
& f_{\mathrm{DE}^{-}}(x, y)=(1-y)^{2}(1-x) .
\end{aligned}
$$

Terms proportional to $\left(m_{e} / M_{K}\right)^{2}$ are neglected. The photon energy spectrum in the CM is shown in Fig. 2 with its $\mathrm{IB}, \mathrm{DE}^{+}$, and $\mathrm{DE}^{-}$contributions. ${ }^{3}$ The $\mathrm{DE}$ terms are evaluated with constant $V, A$ coupling and calculated in ChPT at $\mathscr{O}\left(p^{4}\right)$ [24].

$R_{K}$ has been measured very recentely by KLOE and NA62 on samples of about 14,000 K $\rightarrow e v$ events and of about 50,000 $K \rightarrow e v$ events respectively. KLOE also performed a study of the photon spectrum in $K_{e 2 \gamma} \dot{B}$ oth analysis are inclusive of the IB contribution. The DE has been treated differently.

KLOE define the rate $R_{10}$ as:

$$
R_{10}=\Gamma\left(K \rightarrow e v(\gamma), E_{\gamma}<10 \mathrm{MeV}\right) / \Gamma(K \rightarrow \mu v) .
$$

Evaluating the IB spectrum to $\mathscr{O}\left(\alpha_{\mathrm{em}}\right)$ with resummation of leading logarithms, $R_{10}$ includes $93.57 \pm 0.07 \%$ of the IB,

$$
R_{10}=R_{K} \times(0.9357 \pm 0.0007)
$$

The DE contribution in this range is expected to be negligible. However, the event sample used by KLOE to measure $R_{10}$ still contains a small DE contribution, in particular for decays with high electron momentum in the $\mathrm{CM}, p_{e}$.

\footnotetext{
${ }^{2}$ The same arguments apply in principle to $\Gamma(K \rightarrow \mu v)$. However, there is no helicity suppression in this case. IB must be included and DE can be safely neglected.

3“+" and "-" refer to the photon helicity.
} 


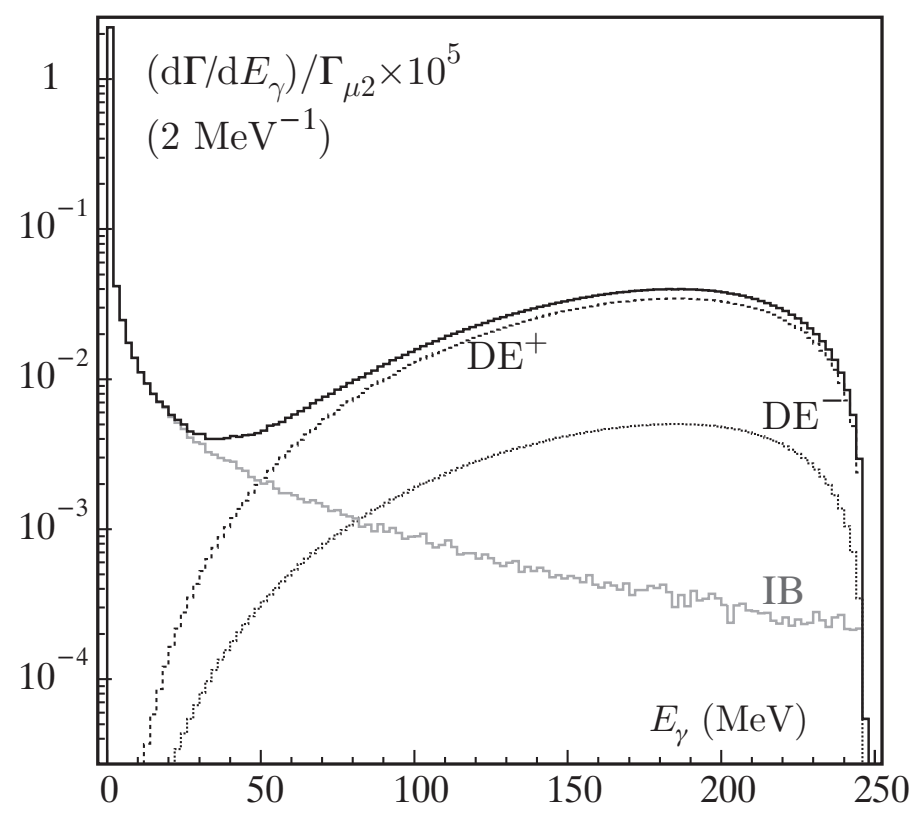

Figure 2: CM photon spectrum for $K_{e 2 \gamma}$ decay. Inner bremsstrahlung (IB) and positive and negative helicity direct emission $\left(\mathrm{DE}^{+}\right.$and $\left.\mathrm{DE}^{-}\right)$contributions are also shown.

In order to subtract this contribution, KLOE has also measured the differential width

$$
\frac{\mathrm{d} R_{\gamma}}{\mathrm{d} E_{\gamma}}=\frac{1}{\Gamma(K \rightarrow \mu \nu)} \frac{\mathrm{d} \Gamma(K \rightarrow e v \gamma)}{\mathrm{d} E_{\gamma}},
$$

for $E_{\gamma}>10 \mathrm{MeV}$ and $p_{e}>200 \mathrm{MeV}$ requiring photon detection, both to test ChPT predictions for the DE terms and to reduce possible systematic uncertainties on the $R_{10}$ measurement.

The DE contribution is strongly rejected (by about a factor of 10) in the NA62 analysis by vetoing non collinear photons. The residual DE is subtracetd according to the KLOE result.

Different approaches have been also employed in discriminating $K \rightarrow e v$ events from the $K \rightarrow \mu \nu$ background ( $10^{5}$ times larger).

The $e / \mu$ separation with calorimeters is more effective at high energy. Therefore, the resulting background rejection factor is about 50 times larger for NA62 than for KLOE. This gap is almost entirely recovered by KLOE exploiting the better kinematics rejection. The resulting effective background contamination is about $14 \%$ in KLOE and $10 \%$ in NA48.

From the kaon and decay particle momenta, $\mathbf{p}_{K}$ and $\mathbf{p}_{\mathrm{d}}$, the squared mass $m_{\ell}^{2}$ of the lepton for the decay $K \rightarrow \ell v$ assuming zero missing mass or the squared missing mass $m_{m i s s}^{2}$ assuming the electron mass for the decay particle can be computed. The distributions of $m_{\ell}^{2}$ and $m_{\text {miss }}^{2}$ are shown in Fig 3 and Fig, 4 for the KLOE and NA62 data, respectively.

The final result from KLOE and the present preliminary result from NA62 are listed in Tab. 1 . Combining these new results with the current PDG value, the new world average reads:

$$
R_{K}=(2.498 \pm 0.014) \times 10^{-5} .
$$

This is in good agreement with the SM expectation [22] and, with a relative error of $0.56 \%$, it is an order of magnitude more precise than the previous world average. 


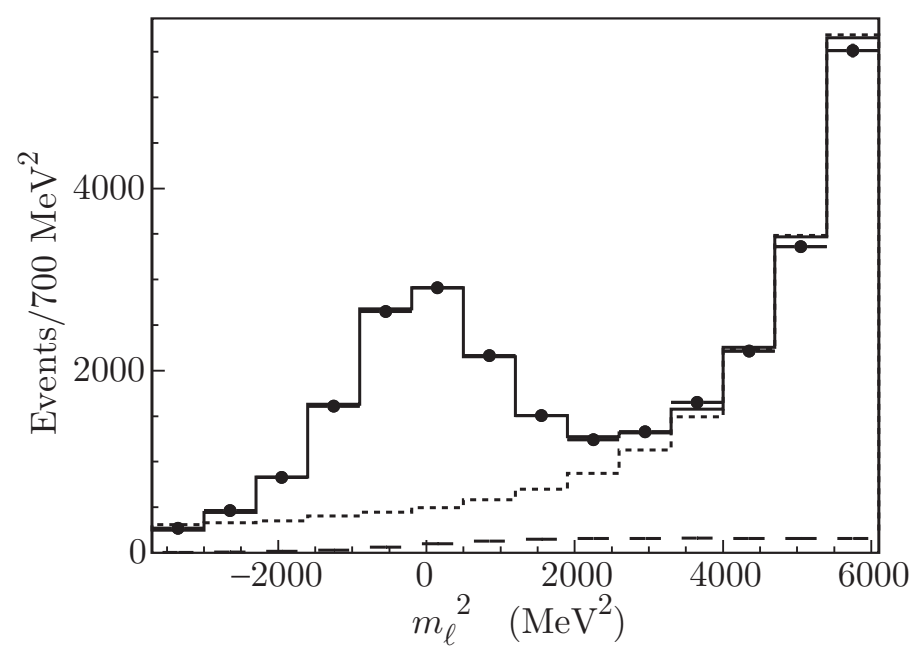

Figure 3: KLOE $K \rightarrow e v$ selection: $m_{\ell}^{2}$ distribution, for data (black dots), MC (solid line), and $K \rightarrow \mu v$ background (dotted line). The contribution from $K \rightarrow e v \gamma$ events with $E_{\gamma}>10 \mathrm{MeV}$ is visible in the left panel (dashed line).

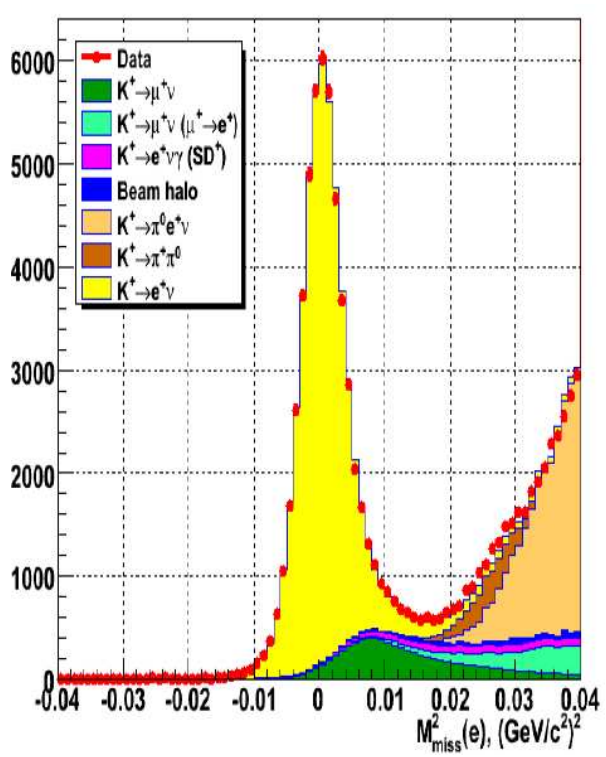

Figure 4: NA62 $K \rightarrow e v$ selection: $m_{m i s s}^{2}$ distribution, for data (red dots), MC signal, and background. The various background contributions are represented by different colours.

The world average result for $R_{K}$ gives strong constraints for $\tan \beta$ and $M_{H^{ \pm}}$, as shown in Fig. 5 . For values of $\Delta_{13} \approx 5 \times 10^{-4}$ and $\tan \beta>50$ the charged Higgs mass is pushed above $1000 \mathrm{GeV} / \mathrm{c}^{2}$ at $95 \% \mathrm{CL}$.

Results on the differential spectrum for the radiative $K \rightarrow e v \gamma$ decay with the condition $p_{e}>$ $200 \mathrm{MeV}$ are given by KLOE, see Table 2, For each $E_{\gamma}$ bin, the integral $\Delta R_{\gamma}$ of $\mathrm{d} R_{\gamma} / \mathrm{d} E_{\gamma}$ over the bin width is measured. In Fig. 6 top, the KLOE measurements are compared to the prediction from $\mathrm{ChPT}$ at $\mathscr{O}\left(p^{4}\right)$ [24] and from the Light Front Quark model (LFQ) of Ref. 25. Integrating over $E_{\gamma}$ 


\begin{tabular}{lc}
\hline \hline & $R_{K}\left[10^{-5}\right]$ \\
\hline PDG & $2.45 \pm 0.11$ \\
NA62 & $2.500 \pm 0.016$ \\
KLOE & $2.493 \pm 0.031$ \\
\hline SM prediction & $2.477 \pm 0.001$ \\
\hline \hline
\end{tabular}

Table 1: Results and prediction for $R_{K}$.

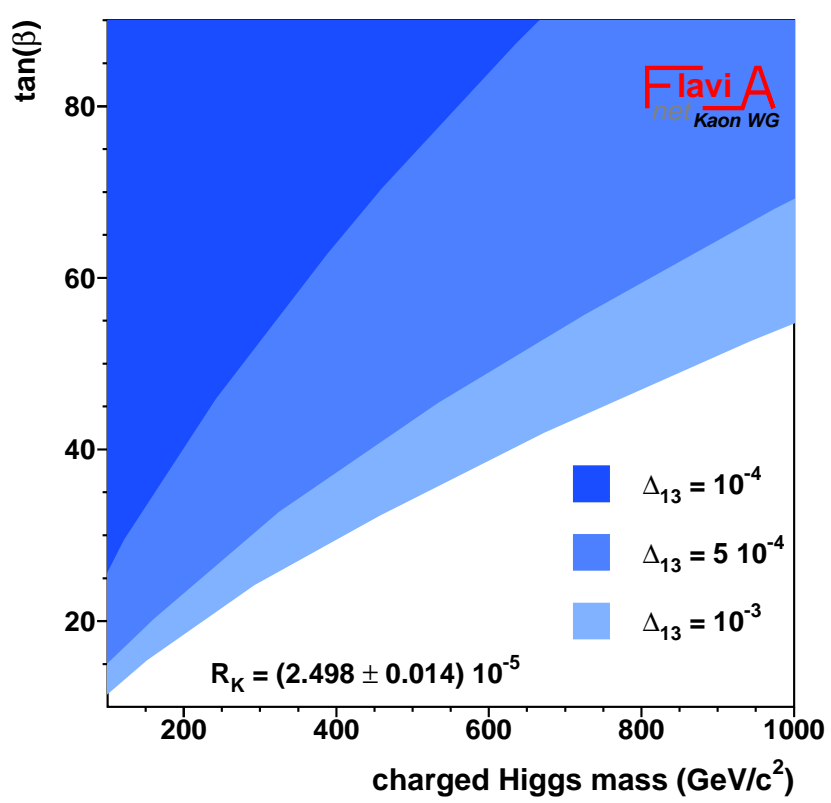

Figure 5: Exclusion limits at $95 \% \mathrm{CL}$ on $\tan \beta$ and the charged Higgs mass $M_{H^{ \pm}}$from $R_{K}$ for different values of $\Delta_{13}$.

from $10 \mathrm{MeV}$ to $250 \mathrm{MeV}$, one obtains:

$$
R_{\gamma}=\left(1.483 \pm 0.066_{\text {stat }} \pm 0.013_{\text {syst }}\right) \times 10^{-5}
$$

in agreement with the prediction $R_{\gamma}=1.447 \times 10^{-5}$ obtained using the values for the effective couplings ( $V$ and $A$ ) from $\mathscr{O}\left(e^{2} p^{4}\right)$ ChPT [24] and using world-average values for all of the other relevant parameters. The $R_{\gamma}$ prediction includes a $1.32(1) \%$ contribution from IB. This result confirms within a $4 \%$ error the amount of DE component in the KLOE MC.

The comparison of the measured spectrum with the ChPT prediction shown in Fig. 6 top suggests the presence of a form factor, giving a dependence of the effective couplings on the transferred momentum, $W^{2}=M_{K}^{2}(1-x)$, as predicted by ChPT at $\mathscr{O}\left(e^{2} p^{6}\right)$ [25]. The form-factor parameters are obtained by fitting the measured $E_{\gamma}$ distribution with the theoretical differential decay width given in Eq. 3.6, with the vector effective coupling expanded at first order in $x$ : $V=V_{0}(1+\lambda(1-x))$. The axial effective coupling $A$ is assumed to be independent on $W$ as pre- 


\begin{tabular}{|c|c|c|}
\hline$E_{\gamma}(\mathrm{MeV})$ & $\varepsilon(\mathrm{e} 2) / \varepsilon(\mu 2)$ & $\Delta R_{\gamma}\left(10^{-6}\right)$ \\
\hline 10 to 50 & $0.104 \pm 0.003$ & $0.94 \pm 0.30 \pm 0.03$ \\
50 to 100 & $0.192 \pm 0.001$ & $2.03 \pm 0.22 \pm 0.02$ \\
100 to 150 & $0.184 \pm 0.001$ & $4.47 \pm 0.30 \pm 0.03$ \\
150 to 200 & $0.183 \pm 0.001$ & $4.81 \pm 0.37 \pm 0.04$ \\
200 to 250 & $0.174 \pm 0.002$ & $2.58 \pm 0.26 \pm 0.03$ \\
\hline
\end{tabular}

Table 2: Results for the integral of $\mathrm{d} R_{\gamma} / \mathrm{d} E_{\gamma}$ on the listed bin widths. Most of the efficiency ratio error is common to all energy bins.

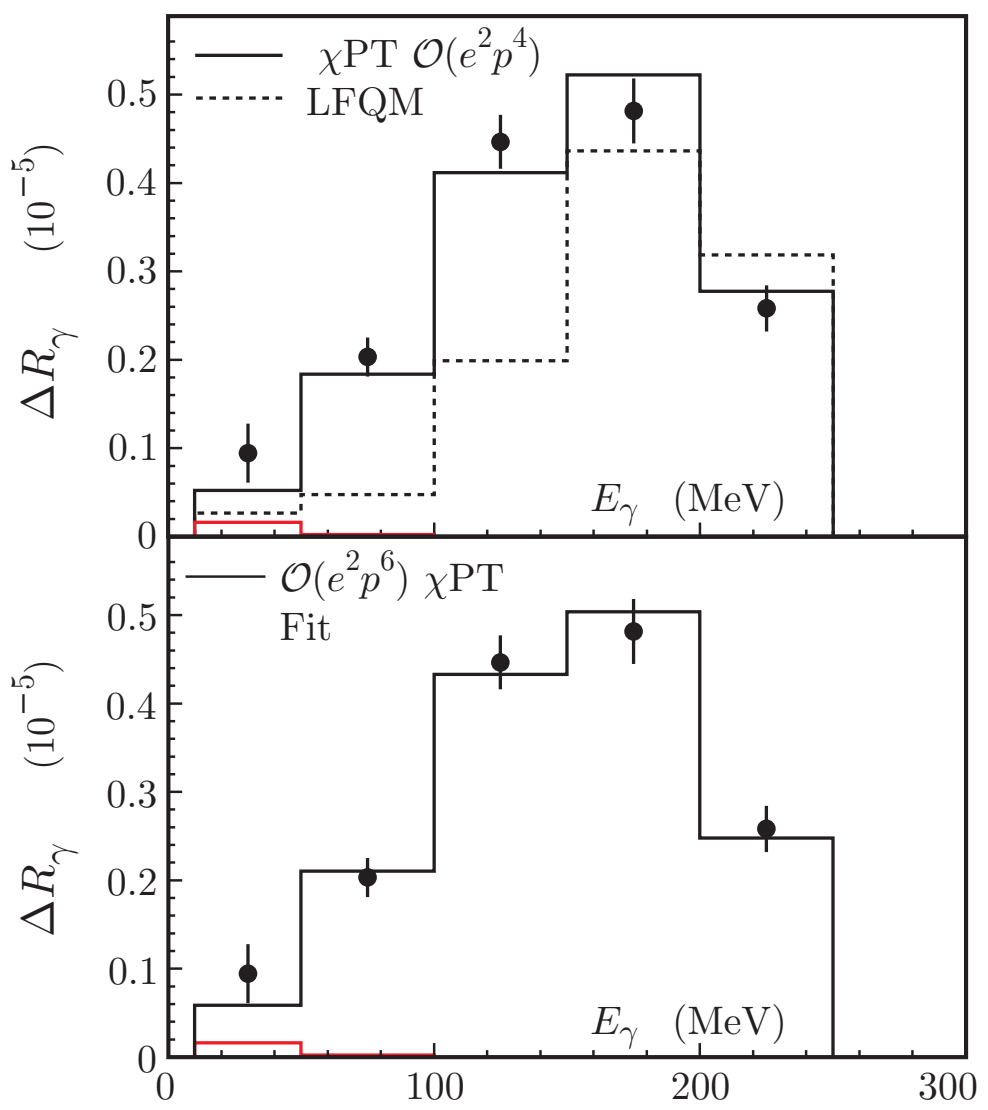

Figure 6: $\Delta R_{\gamma}=\left[1 / \Gamma\left(K_{\mu 2}\right)\right] \times\left[\mathrm{d} \Gamma\left(K_{e 2 \gamma} / \mathrm{d} E_{\gamma}\right]\right.$ vs $E_{\gamma}$. On top data (black dots) are compared to ChPT predictions at $\mathscr{O}\left(e^{2} p^{4}\right)$ and to the LFQ model, see text. At the bottom data are fitted to ChPT at $\mathscr{O}\left(e^{2} p^{6}\right)$. The IB contribution is shown (red line).

dicted by ChPT at $\mathscr{O}\left(e^{2} p^{6}\right)$ [25]. The small contribution from $\mathrm{DE}^{-}$transition to the selected events does not allow a fit to the related $V-A$ component. Therefore, in the fit $V_{0}-A$ is kept fixed at the expectation from ChPT at $O\left(e^{2} p^{4}\right)$, while $V_{0}+A$ and $\lambda$ are the free parameters. The result of this fit is shown in Fig. 6 bottom. KLOE obtains:

$$
\begin{aligned}
V_{0}+A & =0.125 \pm 0.007_{\text {stat }} \pm 0.001_{\text {syst }}, \\
\lambda & =0.38 \pm 0.20_{\text {stat }} \pm 0.02_{\text {syst }},
\end{aligned}
$$


with a correlation of -0.93 and a $\chi^{2} /$ ndof $=1.97 / 3$. This result confirms at $\sim 2 \sigma$ the presence of a slope in the vector form factor, in agreement with the value from ChPT at $O\left(e^{2} p^{6}\right), \lambda \sim 0.4$.

\section{References}

[1] V. Weisskopf and E.P. Wigner, Z. Phys. 63 (1930) 54;

[2] C. Amsler et al. [Particle Data Group], Phys. Lett. B 667 (2008) 1.

[3] J. H. Christenson, J. W. Cronin, V. L. Fitch, R. Turlay, Phys. Rev. Lett. 13 (1964) 138.

[4] F. Ambrosino et al. [KLOE Collaboration], Phys. Lett. B 638 (2006) 140 [arXiv:hep-ex/0603041].

[5] KTeV Collaboration, T. Alexopolous, et al., Phys. Rev. D 70 (2004) 092006.

[6] A. Lai et al. [NA48 Collaboration], Phys. Lett. B 645 (2007) 26 [arXiv:hep-ex/0611052].

[7] M. Antonelli et al. [FlaviaNet Working Group on Kaon Decays], arXiv:0801.1817 [hep-ph].

[8] Particle Data Group, S. Eidelman, et al., Phys. Lett. B 592 (2004) 1.

[9] N. Cabibbo, Phys. Rev. Lett. 10 (1963) 531.

[10] M. Kobayashi, T. Maskawa, Prog. Theor. Phys. 49 (1973) 652.

[11] T. Inami and C. S. Lim, Prog. Theor. Phys. 65 (1981) 297 [Erratum-ibid. 65 (1981) 1772].

[12] S. Herrlich and U. Nierste, Nucl. Phys. B 419 (1994) 292 [arXiv:hep-ph/9310311].

[13] A. J. Buras, M. Jamin and P. H. Weisz, Nucl. Phys. B 347 (1990) 491.

[14] S. Herrlich and U. Nierste, Nucl. Phys. B 476 (1996) 27 [arXiv:hep-ph/9604330].

[15] D. J. Antonio et al. [RBC Collaboration and UKQCD Collaboration], Phys. Rev. Lett. 100 (2008) 032001 [arXiv:hep-ph/0702042].

[16] C. Aubin, J. Laiho and R. S. Van de Water, arXiv:0905.3947 [hep-lat].

[17] G. Isidori and P. Paradisi, Phys. Lett. B 639 (2006) 499 [arXiv:hep-ph/0605012]; W. S. Hou, Phys. Rev. D 48, 2342 (1993); A. G. Akeroyd and S. Recksiegel, J. Phys. G 29, 2311 (2003) [arXiv:hep-ph/0306037].

[18] G. Isidori and A. Retico, JHEP 0111, 001 (2001) [arXiv:hep-ph/0110121].

[19] A. Masiero, P. Paradisi and R. Petronzio, Phys. Rev. D 74 (2006) 011701 [arXiv:hep-ph/0511289].

[20] K. Ikado et al., Phys. Rev. Lett. 97, 251802 (2006) [arXiv:hep-ex/0604018]; B. Aubert et al. [BABAR Collaboration], Phys. Rev. D 76, 052002 (2007) [arXiv:0708.2260 [hep-ex]].

[21] W.J. Marciano and A. Sirlin, Phys. Rev. Lett. 71 (1993) 3629; M. Finkemeier, Phys. Lett. B 387 (1996) 391.

[22] V. Cirigliano and I. Rosell, Phys. Rev. Lett. 99 (2007) 231801.

[23] A. Masiero, P. Paradisi and R. Petronzio, JHEP 0811 (2008) 042

[24] J. Bijnens, G. Colangelo, G. Ecker and J. Gasser, arXiv:hep-ph/9411311. Published in 2nd DAPHNE Physics Handbook:315-389.

[25] C.H. Chen, C.Q. Geng and C.C. Lih, Phys. Rev. D 77 (2008) 014004. 